\title{
A NOVEL APPROACH FOR RECONSTRUCTION OF LARGE ORO-ANTRAL FISTULAE DEFECTS WITH SIMULTANEOUS SINUS LIFT. (A CLINICAL STUDY)
}

\author{
Hesham El-Hawary* and Amr Gibaly**
}

\begin{abstract}
Background: The Oro-antral fistulae (OAF) compromises the oral mucosa and the alveolar bone integrity, depleting its capacity to receive dental implants.

Purpose: This clinical study aims to assess the clinical outcomes and the radiographic vertical bone gain of an innovative technique that combines the (OAF) closure, bone grafting, and sinus lift.

Patients and methods: The study represents a case series conducted on thirteen patients with large (OAF), closed by sliding the buccal advancement flap combined with a distant palatal releasing incision after inverting and elevating the fistulous epithelial tract to set, elevate and conceal the floor of the maxillary sinus from the underlying bone graft. Meanwhile, evaluating the clinical outcomes and justifying a radiographic linear bone height comparison between the sinus floor height preoperatively and three months after.
\end{abstract}

Results: The healing went uneventful, with neither shallowing of the vestibular depth nor obliterating the sinus cavity. The bony sinus floor was admirably elevated, and the grafted defects demonstrated appropriate bone width and height. The mean baseline bone height recorded $(9.28$ $\pm 2.4 \mathrm{~mm})$ versus $(17.54 \pm 1.6 \mathrm{~mm})$ for the three months postoperative. The vertical bone gain ranged from (4 to $12.5 \mathrm{~mm})$, with a mean of $(8.26 \pm 2.6 \mathrm{~mm})$, computing a statistically significant linear gain increase $(\mathrm{p}<0.00001)$.

Conclusion: The novel approach provided comprehensive coverage of the oral defect, an appropriate primary elevation of the maxillary sinus with consolidated alveolar graft, cutting short the routine treatment time with the conduction of significant linear bone height gain values.

KEYWORDS: Oro-antral fistula, Buccal advancement flap, Alveolar bone graft, Maxillary sinus lift, and Radiographic vertical bone gain.

\footnotetext{
* Associate Professor of Oral and Maxillofacial Surgery, Faculty of Dentistry, Cairo University, Egypt.

** Lecturer of Oral and Maxillofacial Surgery, Faculty of Dentistry, Beni-Suef University, Egypt.
} 


\section{INTRODUCTION}

The oro-antral communication (OAC) defects represent a conjunction between the oral cavity and the maxillary air sinus, most commonly evoked by violent teeth extractions. Although small defects may heal spontaneously, untreated moderate and large-sized communications frequently develop into connecting epithelial fistulae that construct a pathologic route for bacterial invasion between the oral cavity and the antrum, precipitating the subsequent maxillary sinusitis. Which demands surgical soft tissue closure, either in solitary, aiming to provide complete soft-tissue coverage or associated with bone grafting of the communication defect to buttress the prospected implant housing. ${ }^{(1)}$

Various surgical techniques had been proposed for the closure of (OAF). The buccal advancement flap, which defined the most common local flap (2), was first introduced by Rehrmann (3) in 1936, who described an elliptical incision that surrounds the fistulous tract prior to its excision along with a crestal incision and two vertical releasing incisions toward the buccal sulcus, composing a trapezoidal buccal advancement flap with profuse blood supply, capable for complete coverage of the underlying bone defect after the horizontal scoring of its periosteum.

Unfortunately, the buccal advancement flap is usually associated with excessive tension that would precipitate flap failure and wound dehiscence and depletes the vestibular depth, limiting the prosthetic restorations' selection. Furthermore, the (OAF) is associated with violated alveolar bone, which defers the defect's rehabilitation as it frequently demands later sinus lifting and secondary bone grafting before the placement of root-form dental implants. ${ }^{(4)}$

Several filling materials were postulated for obliterating the oro-antral defect, including harvesting autogenous bones ${ }^{(5)}$, cartilaginous grafts ${ }^{(6)}$, and introducing xenografts, alloplasts, or synthetic materials. ${ }^{(7)}$ Although the autogenous bone graft represents the gold standard, it carries out an adjoint donor site morbidity and volumetric unpredictability. (8) Schmitt et al. (9) in 2015 compared the sole augmentation of Xenogenic bone versus augmenting an equal mix proportion of autogenous and Xenogenic bone graft for maxillary sinus elevation and reported insignificant histomorphometric difference between the bone quality of both grafts; meanwhile, the later insertion of the dental implants.

The authors of this clinical study are aiming to assess the radiographic vertical bone gain and the clinical outcomes of an innovative surgical approach that combines soft tissue closure, bone grafting, and immediate sinus lifting after sliding the buccal advancement flap combined with a distant palatal releasing incision. Looking forward to releasing the flap tension and ensuring direct augmentation and elevation of the maxillary sinus floor.

\section{PATIENTS AND METHODS}

\section{Criteria of patient selection}

Thirteen patients, nine males, and four females ranging in age from (23 to 50) years old, with unilateral chronic (OAF), were incorporated into this study. The inclusion criteria demanded that all the selected patients would have a history of violent extraction of either the first or the second maxillary molar of at least one month before the clinical presentation, with failure of healing of the antral communication and the subsequent formation of (OAF) with a minimum diameter of four millimeters of the outer bony defect. (Table 1) Considering that a retained root is neither confined within the alveolar bone nor the maxillary air sinus. Meanwhile, the smoker patients and those with sinus pathosis or systemic chronic diseases that would compromise the maxillary sinus membrane's integrity or normal bone healing were excluded. The authors recorded a comprehensive history, including detailed medical and dental histories from the patients, who obtained 
digital panoramic radiographs that represented a primary survey for assessing the presence of retained roots or pathological entities (Figure: 1 A). The selected patients were further submitted to Cone Beam Computed Tomography (CBCT); (Planmeca, ProMax 3 D, Helsinki, Finland) that establish a fixed reference for identifying the exact hight of the maxillary sinus bony floor preoperatively and precisely calibrated the outer diameter of the fistulous bone defect (Figures $1 B \& \mathbf{C}$ ). Which was further assessed by clinical calibration of the bone defect utilizing a periodontal probe verification under local anesthesia (L.A.)

The selected patients were subjected to a preoperative protocol to control maxillary sinusitis, nasal congestion, and discharge. Eradication of the infection was carried out for one week before surgery; daily saline irrigation was accomplished, coupled with attempting pus evacuation. The patients administrated Clindamycin, $300 \mathrm{mg} / 8 \mathrm{~h}$ (Dalacin-C, Pfizer, Egypt) and Antihestamininc, $120 \mathrm{mg} / 12 \mathrm{~h}$ (Telfast, Sanovi, Egypt), NSAID, $600 \mathbf{m g} / \mathbf{8} \boldsymbol{h}$ (Ibobrufen, El-Kahira/Abbott. Egypt). And nasal decongestant spray (Aphrin; Bayer, Fl., U.S.A.) for one week before surgery.

TABLE (1): The Demographic and clinical data.

\begin{tabular}{|c|c|c|c|c|}
\hline & Gender & $\begin{array}{c}\text { Age } \\
\text { (years) }\end{array}$ & Tooth\# & $\begin{array}{c}\text { Mesio-Destal dimension } \\
\text { of the OAF }(\mathrm{mm})\end{array}$ \\
\hline 1 & Female & 35 & 14 & 9.2 \\
\hline 2 & Male & 43 & 15 & 4.2 \\
\hline 3 & Female & 27 & 3 & 7.3 \\
\hline 4 & Male & 23 & 2 & 6.3 \\
\hline 5 & Male & 29 & 2 & 7.4 \\
\hline 6 & Male & 49 & 14 & 5.2 \\
\hline 7 & Female & 35 & 3 & 8.3 \\
\hline 8 & Male & 41 & 2 & 7.2 \\
\hline 9 & Male & 48 & 15 & 4.8 \\
\hline 10 & Male & 31 & 3 & 4.9 \\
\hline 11 & Female & 42 & 14 & 9.5 \\
\hline 12 & Male & 50 & 14 & 6.3 \\
\hline 13 & Male & 38 & 2 & 8.4 \\
\hline mean & & 37.8 & & 6.8 \\
\hline $\begin{array}{l}\text { St. } \\
\text { dev }\end{array}$ & & \pm 8.4 & & \pm 1.7 \\
\hline
\end{tabular}

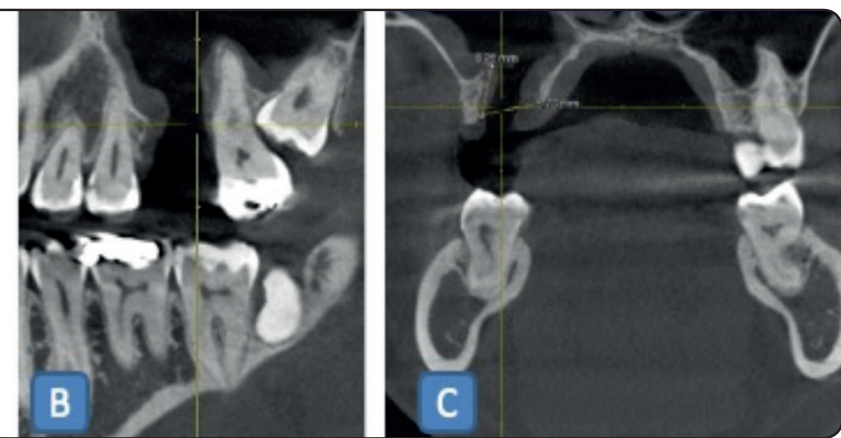

Fig. (1) (A): Preoperative screening digital panoramic radiograph.(B): Preoperative sagittal CBCT view with measuring the OAC height and width. (C): Preoperative coronal CBCT view. 


\section{Operative procedure}

(Diagram 1-8):

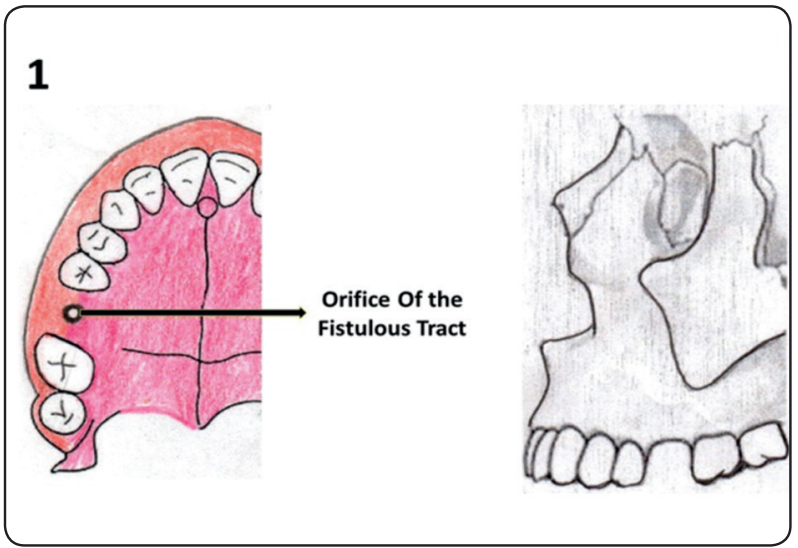

Diagram (1): The fistulous orifice.

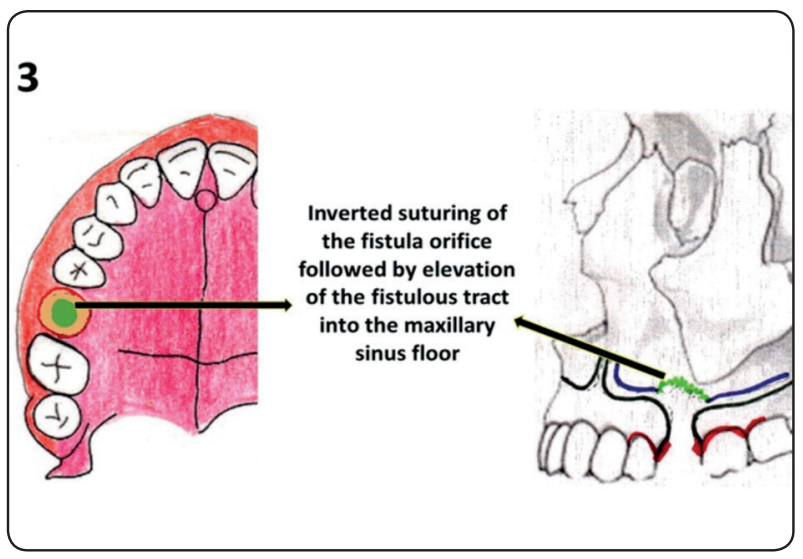

Diagram (3): Suturing of the fistulous epithelium and elevating it from the fistulous tract toward the sinus.

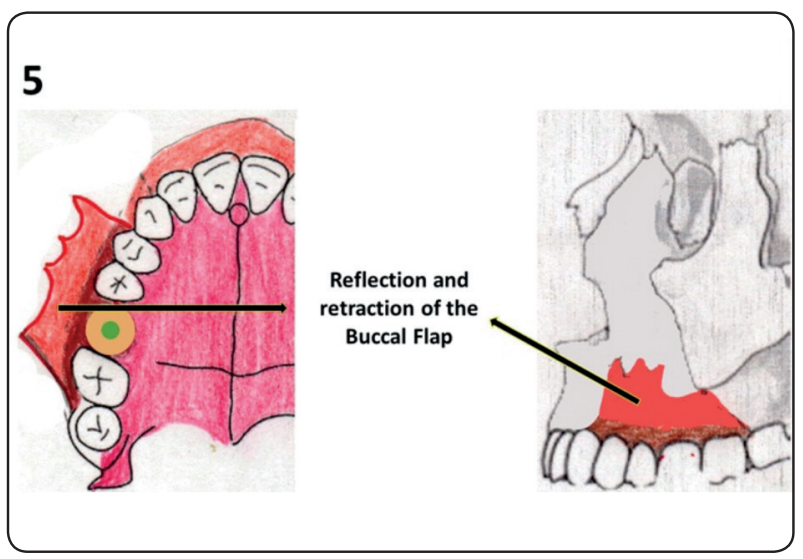

Diagram (5): The reflection of the buccal flap.

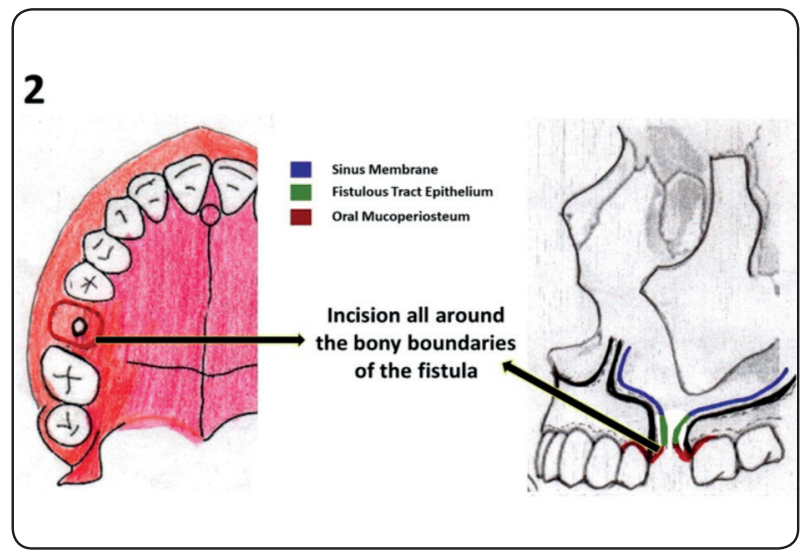

Diagram (2): Incision of the fistulous tract resting on the sound bone.

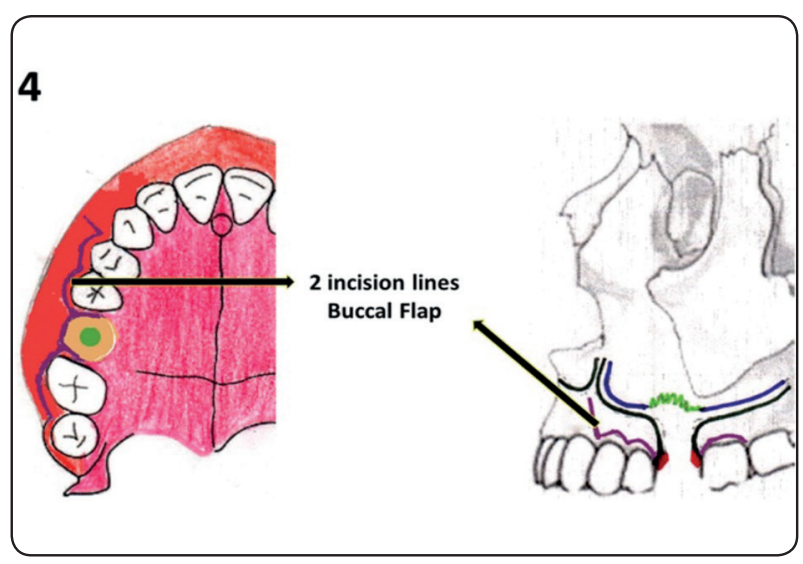

Diagram (4) The two-incision line buccal flap.

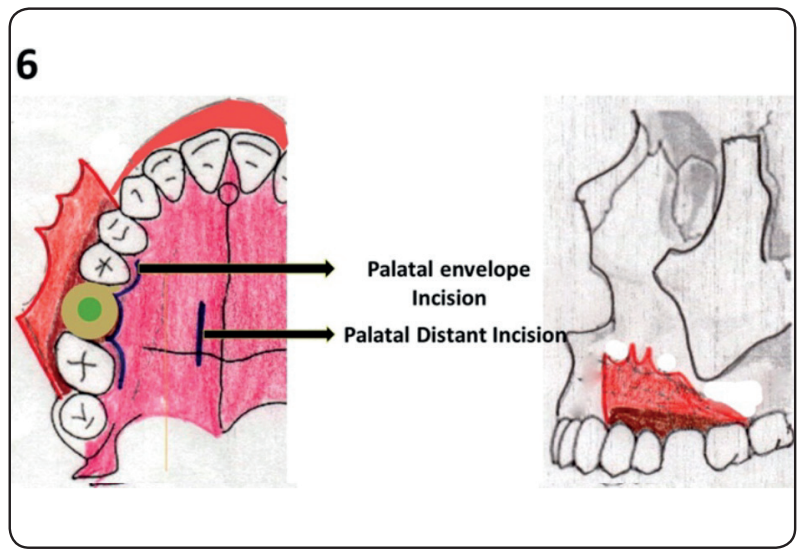

Diagram (6): The palatal incisions. 


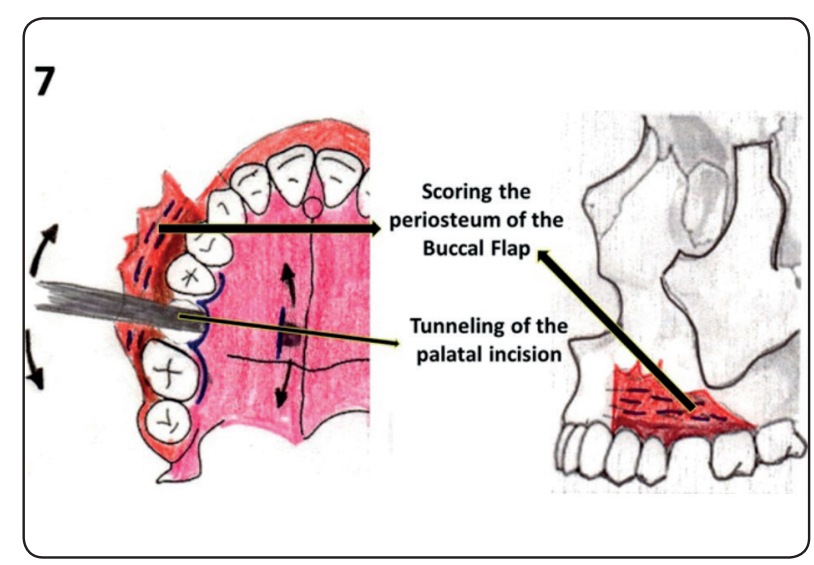

Diagram (7): Scoring of the buccal flap and tunneling the palatal incision.

All the surgical procedures were carried out under Local anesthesia (Articaine 40mg/1ml, epinephrine 1:100.000. Artinibsa, Spain) through buccal and palatal infiltration.

\section{I) The elevation of the fistulous tract}

The fistulous boundaries were then probed aided by a sharp periodontal probe to allocate the (OAC) bony edges. Using a Bard parker blade \#15, an elliptical incision is sculptured around the bone defect resting on the sound bony walls, incising the fistulous epithelium from the surrounding oral mucoperiosteum (Diagram 2, Fig 2A). Then the edges of the fistulous epithelium are everted upward, towards the maxillary sinus, and the fistulous epithelium is then sutured using inverted suturing technique by Vicryl 0000 (AssuCryl, Assut, Switzerland) and elevated in an upward direction by sinus elevators in a procedure similar to that executed for sinus membrane lifting (Diagram

\section{3, Figures $2 B \& 2 C$ ).}

\section{II) The buccal advancement flap}

The trapezoidal flap utilizes a gingival incision, which adjoins the incised fistulous tract and extends for two teeth anteriorly and one tooth posteriorly. The vertical incision is initiated anteriorly and extended upward in an obtuse-angular direction,

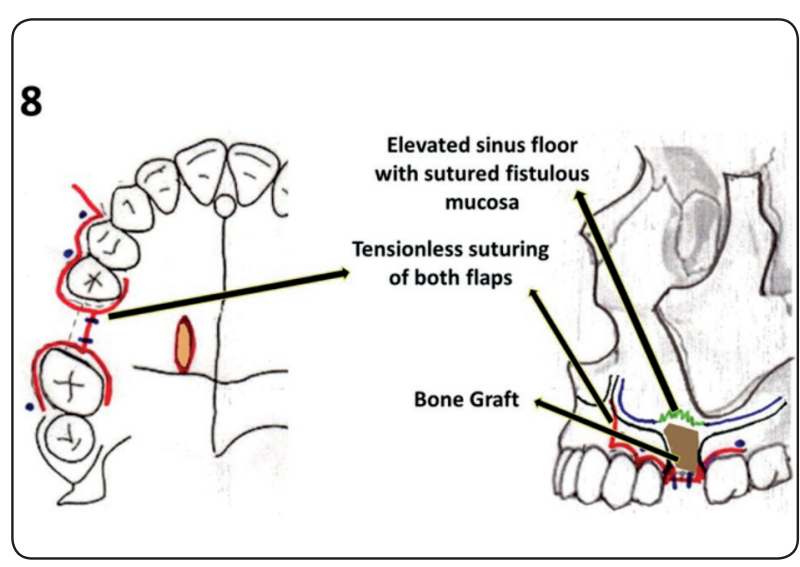

Diagram (8): Insertion of bone graft and primary tensionless closure with the distant palatal incision expansion.

incorporating the dental papillae anteriorly and posteriorly. The flap is then reflected and retracted upward in a usual manner, followed by horizontal scoring of the periosteum to allow for unrestricted advancement of the flap (Figure 3A, Diagrams $4 \& 5)$.

\section{III) The palatal mucoperiosteum modification}

$1 \mathrm{Cm}$ horizontal palatal incision is sculptured opposite the fistula and parallel to the median palatine suture, positioned $5 \mathrm{~mm}$ apart to avoid injuring the greater palatine artery (Diagram 6, Figure 3B). Aided by a mucoperiosteal elevator, a tunnel is created between the palatal mucoperiosteum and the underlying palatal bones along the fistulous tract, which connects the defect with the palatal incision and frees the palatal mucoperiosteum. Followed by the wound toilet and copious saline irrigation (Diagram 7).

\section{IV) Bone grafting, flap transposition, and suturing}

The communication gap is then obliterated by Xenogenic bone (Granular bone xenograft; Geistlich Bio-OSS, Switzerland) and condensed upward. The Xenogenic bone's gradual compaction further elevates the fistulous tract and secures the overlying Schneiderian membrane's consequent elevation (Diagram 8). 
The buccal flap was then slid, suturing its advanced edges to the approximated free palatal flap, which was transported to cover the bone defect with minimal tension after releasing the distant palatal incision. At the middle of the vertical incisions, a suture is gently placed to cover the bare bone over the slide vertical flap margins and fill the papillary area (Diagram 8 , Figure $3 C$ ).

\section{Postoperative care}

Postoperatively, the patients continued the same preoperative medication regimen for five days, instructed for maintaining proper oral hygiene, a soft diet, and nasal precaution measurements (avoiding nasal sneezing, nose-blowing, and exerting negative pressure). The authors instructed the patients to immediately accomplish postoperative panoramic radiographs to verify the Schneiderian membrane's surgical integrity by inspecting the sinus cavity's radiolucent clearance (Figure 4A). By the end of the three -month follow-up period, all the patients were subjected to (CBCT) (Figures $4 B \& 4 C$ ) to appraise the surgical intervention and calibrate the vertical bone gain.

\section{Radiographic standardization, superimposition, and digital interpretation}

The preoperative and the three months postoperative CBCTs were submitted for all the patients utilizing the same machine and same radiographic exposure parameters (Planmeca, ProMax 3-D, Helsinki, Finland; $15 \mathrm{~mA}$ and $85 \mathrm{KV}$ ), which defined

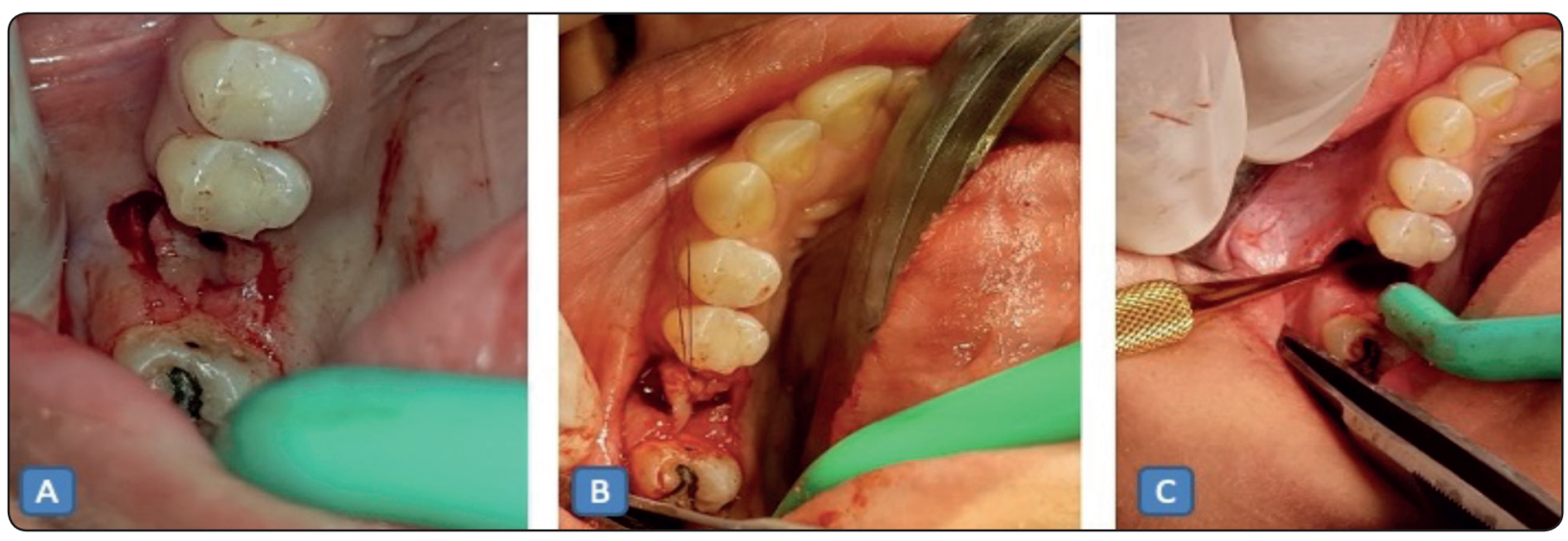

Fig. (2): (A): Incision all around the bony edges of the fistulous tract. (B) : Inverted Suturing of the edges of the fistula. (C) The fistulous tract is dissected from the bony walls, and the sinus floor is lifted using sinus lift elevators.
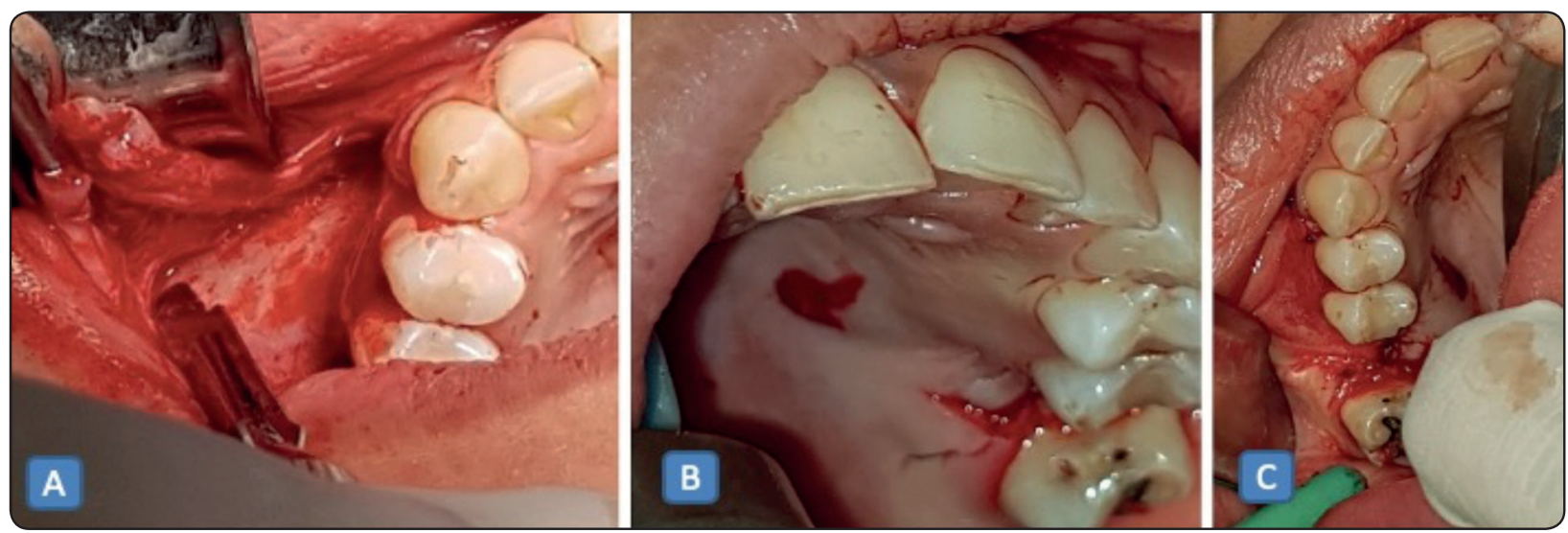

Fig. (3): (A): Scoring the periosteum of the retracted buccal flap. (B): The Palatal Distant incision. (C): Closure of both flaps showing the expansion in the distant palatal incision. 
a preoperative fixed baseline as well as the final postoperative inquiry for justification the radiographic comparison. After importing the selected sagittal radiographic views to DICOM (Digital Imaging and Communications in Medicine) files, which introduced to the software (MIMICS medical 21.0 Materialise. Leuven, Belgium), the vertical bone height was calibrated from the highest horizontal level of the bony floor of the maxillary sinus, perpendicular to the corresponding point on the alveolar bone crest. The allocation of the preoperative violated maxillary sinus floor was determined by plotting a horizontal line that connected the mesial and distal margins of the perforation, which considered the preoperative radiographic height of its bony floor. On the other hand, the postoperative radiographic bone height utilized simple marking of the clear bone floor. The linear calibration of both hights represented the preoperative and postoperative bone hights, and the difference between them represented the bone hight gain (Figures 5 A \& 5 B)

The authors activated the preoperative and postoperative CT scans' digital superimposition on (Adobe Photoshop, version 22.1.1. Adobe, Microsoft Corporation, USA) for the illustrated case in (Figure 6) to demonstrate the radiographic outcome of the surgical intervention.
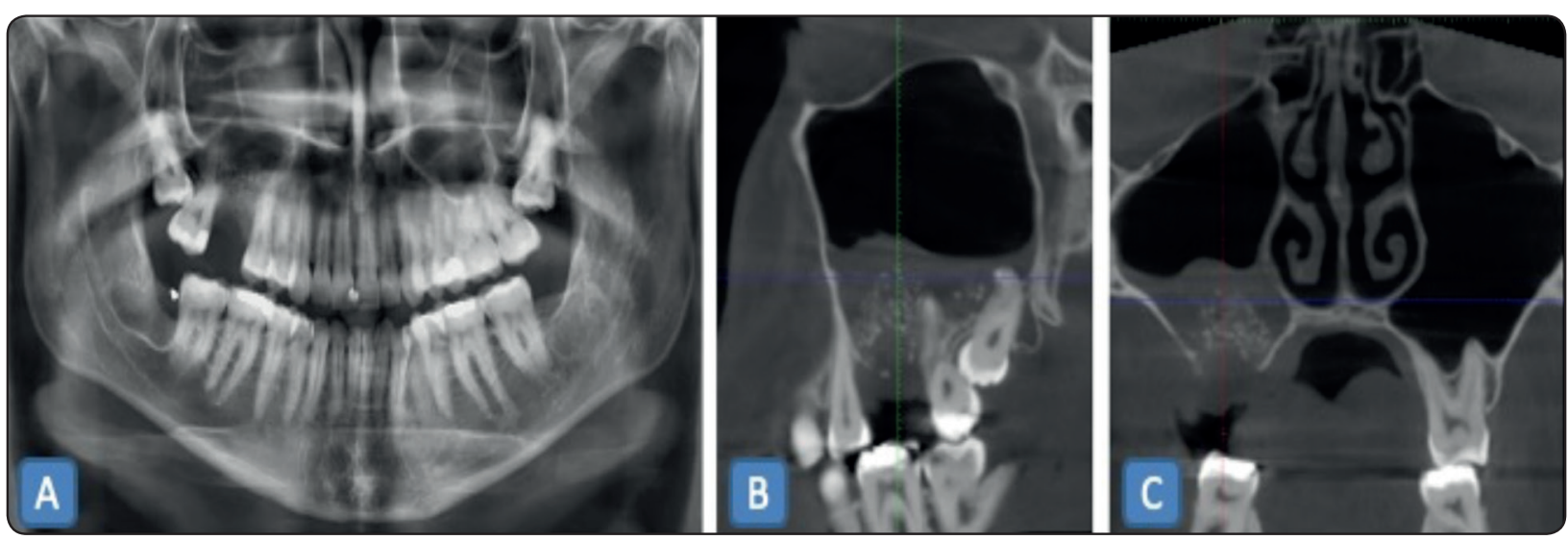

Fig. (4): (A): Immediate Postoperative digital panoramic radiograph. (B): Three months postoperative sagittal CBCT view, measuring the bone height $(\mathrm{C})$ : Three months postoperative coronal CBCT view.
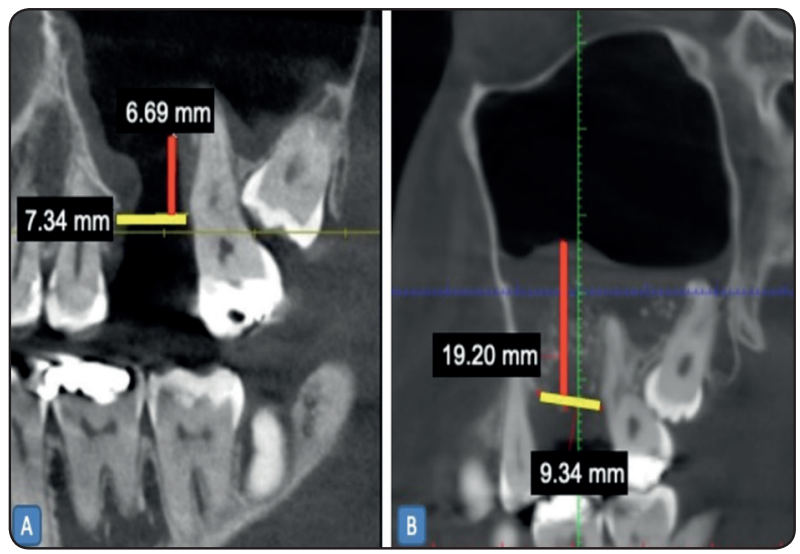

Fig. (5) : (A): Preoperative sagittal CBCT view with measuring the OAC height and width. (B): Three months postoperative sagittal CBCT view, measuring the gained bone height.

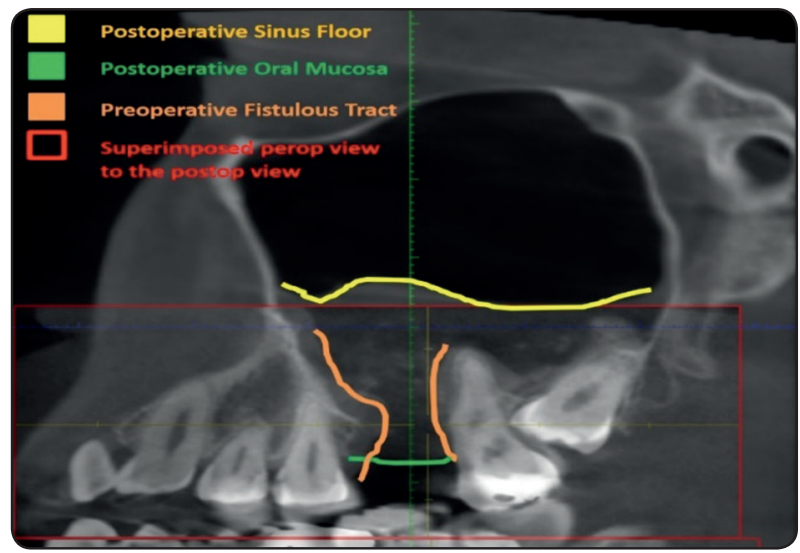

Fig. (6): Superimposition of the preoperative and the postoperative CBCT. 


\section{Statistical analysis}

The statistical analysis implemented data analysis using IBM SPSS. (Statistical Package for Social Sciences, Version 22. SPSS Inc., Chicago, IL). The numerical data were described as means and standard deviations, which examined for normality using the Kolmogrov-Smirnov test. Relieving that the distribution was normal, the student t-test transacted the comparisons between the mean preoperative bone height and the three months postoperative, the p-value; $p<0.05$ considered statistically significant

\section{RESULTS}

The current study was conducted on thirteen patients of both sexes, with a mean age of (37.8 \pm 8.3 years) who demonstrated (OAF) with a mean Mesio-Destal dimension of $(6.85 \pm 1.7 \mathrm{~mm})$, with at least two months of neglected treatment after violent extraction of a first or second maxillary molar. All the patients expressed the signs and symptoms of maxillary sinus infection. However, the preoperative treatment protocol alleviated the clinical symptoms and irradicated the maxillary sinus infection among all the patients before surgery.

\section{Clinical findings}

Intraoperatively, the surgical elevation of the buccal and palatal flaps went smooth through all of the cases. Both flaps were appropriately advanced and provided adequate unstretched complete soft tissue cover for the bony defects. Furthermore, the buccal flap vertical edges adequately stretched and covered the marginal bare bone. On the other hand, the fistulous tract dissection and elevation was troublesome in three cases, where one tract was partially lacerated, upon being sutured but successful inverted suturing and elevation was adopted, and two epithelial tracts were tightly attached to the surrounding bone and completely damaged meanwhile being separated; hence they were excised and replaced with collagen membranes (Geistlich Bio-Gide, Switzerland)

The healing went uneventful through all the communication defects. However, three patients exhibited significant postoperative pain and swelling. The surgically reconstructed defects neither revealed postoperative infection and wound dehiscence nor graft rejection through the healing phase. After resolving the postsurgical phase's signs, none of the patients exhibited maxillary sinusitis nor excessive shallowing of the vestibular depth (Figure 7). The immediate postoperative panoramic views judged the inversion and lifting of the fistulous tract admirable, as all the maxillary sinus cavities were radiographically clear, and the concealing inquiry obviously framed the radiopaque graft (Figure 4A).

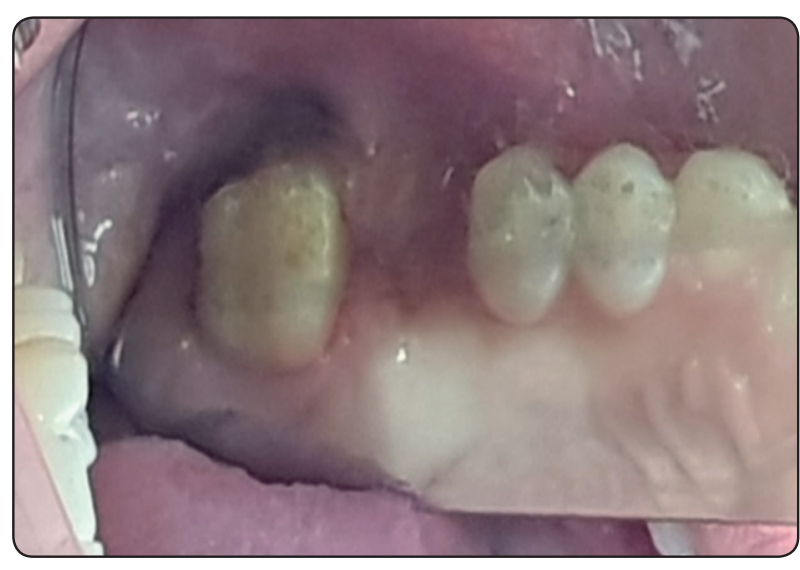

Fig. (7): One-month postoperative clinical photograph showing perfect healing of the soft tissue with excess malleability of the buccal vestibule.

\section{Radiographic Findings}

The C.B.C.T sagittal and coronal cuts three months after bone grafting and sinus lift demonstrated that the bony sinus floor was definitely elevated, and the compacted graft completely obliterated the communicating defects. Moreover, all the grafted defects demonstrated appropriate alveolar bone width and height to accommodate suitable size implant fixtures. (Figures $4 B \& 4 C$ ). 


\section{The radiographic bone height gain}

The mean baseline bone height for the violated sinus floors (Preoperative bone height) was recorded $(9.28 \pm 2.4 \mathrm{~mm})$. The three months postoperative bone height was recorded $(17.54 \pm 1.6 \mathrm{~mm})$. The height of the vertical bone gain ranged from (4 to $12.5 \mathrm{~mm})$, with a mean of $(8.26 \pm 2.6))$ Table 2$)$. The student t-test transaction between the mean preoperative bone height and the three months postoperatively counted statistically significant linear bone height gain increase; $(\mathrm{p}<0.00001)$.

TABLE (2): The bone height gain values:

\begin{tabular}{|c|c|c|c|}
\hline & $\begin{array}{l}\text { Height of the } \\
\text { preoperative } \\
\text { sinus bony floor } \\
\text { (mm) }\end{array}$ & $\begin{array}{l}\text { Height of the } \\
\text { postoperative } \\
\text { sinus bony floor } \\
\text { (mm) }\end{array}$ & $\begin{array}{c}\text { Bone height } \\
\text { gain values } \\
(\mathrm{mm})\end{array}$ \\
\hline & 11 & 15.5 & 4.5 \\
\hline & 7 & 18.9 & 11.9 \\
\hline & 6.7 & 19.2 & 12.5 \\
\hline & 10.5 & 18 & 7.5 \\
\hline & 12.3 & 20 & 7.7 \\
\hline & 8.3 & 14.3 & 6 \\
\hline & 11.4 & 18.9 & 7.5 \\
\hline & 6.8 & 16.2 & 9.4 \\
\hline & 7.3 & 14 & 6.7 \\
\hline & 13.6 & 17.6 & 4 \\
\hline & 8.2 & 18.4 & 10.2 \\
\hline & 6.2 & 18.1 & 11.9 \\
\hline & 11.4 & 19 & 7.6 \\
\hline Mean & 9.3 & 17.5 & 8.3 \\
\hline St. dev. & \pm 2.4 & \pm 1.9 & \pm 2.6 \\
\hline
\end{tabular}

$p<0.05$

\section{DISCUSSION}

The wide current use of dental implants would announce alveolar bone grafting of the communication defects a routine. The defect's complex nature compromises the mucoperiosteal oral mucosa, the alveolar bone integrity, and the maxillary sinus floor's concealment. This demands relaxed watertight soft tissue closure of the oral mucose, establishing a concrete alveolar bone graft underneath the lifted sinus floor to restore the native capacity of the maxillary alveolar bone.

Saleh and Issa ${ }^{(10)}$ represented that the proper blood perfusion and the direct access to the bony defect accomplished by the broad trapezoidal designed buccal advancement flap allow for rapid healing and efficient debridement and eradication of infection. The anterior and posterior vertical releasing incisions, coupled with the periosteal scoring, simplifies stretching and adjusting the pedicle to overlay the defect properly. However, the authors accused the flap of excessive tension and undue shallowing of the vestibular depth. This comes in compliance with the authors of the current study, as we believe that the broad coverage of the buccal advancement flap, if accompanied with releasing the flap tension, would be ideal for providing a reliable soft tissue cover for moderatesized (OAF) even if located more palatal. Hence, the authors designed sliding of the buccal advancement flap, along with the distant palatal releasing incision, which obviously extended the expanse of soft tissue coverage, which would contribute to the uneventful flap healing, recorded among all the study cases and the usual texture of the formed mucosa covering the underlying bone graft and the elevated fistulous tract, which deems essential for providing an acceptable biological mucosal width and favorable emergence profiles for the proposed root form dental implants.

Although Abuabara et al. ${ }^{(11)}$ in 2006 assumed the fistulous tract necrotic and considered its debridement essential for obtaining normal healing as well 
as to avoid maxillary sinusitis and graft rejection, the authors of this study believe that the epithelial tract attains a sort of vitality through its firm adhesion to the surrounding bone and soft tissues, proven by the presence of bleeding points in the tract upon probing. Preserving the tract vascularity was retained by persisting its integrity to the bone defect's superior edges. The authors also attribute the success of the sinus lifting to the dense nature of the fistulous tract that properly concealed both the maxillary sinus and the Xenogenic bone graft and sustained the relocating pressure of graft compaction.

Coinciding with Lee ${ }^{(12)}$, who attributed the high failure rates of the combined sinus lifting with the regular (OAF) closure to the difficulty of eradicating the infection simultaneous with restoring the integrity of the injured sinus floor, the present study results followed a strict preoperative control of infection along with fashioning the epithelial tract to separate the sinus cavity from the underlying graft. Which successfully eradicated the co-existent infection and sealed the graft, avoiding its communication with the maxillary sinus.

The three-month postoperative radiographic analysis demonstrated that lifting of the fistulous epithelial, followed by the gradual packing and condensation of the Xenogenic bone graft, conformed to a beneficent reconstruction of the communication defects quantitatively. This agrees with Ogunsalu ${ }^{(13)}$, who demonstrated that a (BIOOSS) compaction beneath resorbable membranes generated a consolidated graft that restored the communication defect and buttressed the inserted dental implants.

Matching what was reported by Hising et al. ${ }^{(14),}$ who recorded intense bone regeneration after Xenogenic augmentation of the elevated sinus membranes, the current study attributes part of the significant vertical bone gain to osteoconductive properties and volumetric stability of the Xenogenic bone graft. At the same time, contributing the other part to the crafted space, maintained by the fashioned epithelial tract, which coincides with Sohn et al. ${ }^{(15,16)}$, who defined that securing the enclosure of the created space beneath the elevated sinus floor amounts to an osteogenic capacity of that of the bone graft.

In this study, the amount of vertical bone height gain ranged between ( 4 and $12.5 \mathrm{~mm}$ ), with a mean of (8.26 \pm 2.6$)$, which seems comparable to the vertical bone gain range of $(6 \mathrm{~mm}$ to $10 \mathrm{~mm})$ and a mean of $(8.6 \mathrm{~mm})$ recorded by Kilin et al. ${ }^{(17)}$ in 2018 after grafting the sinus floor with an equal mixture of autogenous cancellous particulates and Xenograft utilizing an open lateral window technique. This yields the results of this study proportionate with those of the standard technique.

\section{CONCLUSION}

The novel approach; described in this study defined an appropriate elevation of the maxillary sinus, fair consolidation of the alveolar bone graft, provided comprehensive unstressed coverage of the oral defect, and cut short the routine treatment time, as the primary elevation of the maxillary sinus floor and alveolar bone grafting together with the soft tissue closure had conceded feasible. Furthermore, conducted linear bone gain height values comparable to the side-window open sinus lift.

\section{List of abbreviations :}

OAC: Oro-antral comminucation

$\boldsymbol{O A F}$ : Oro-antral fistula

CBCT: Cone Beam Computed Tomography

\section{LA: Local anesthesia}

\section{Conflict-of-interest notification:}

The authors declare that they have no conflict of interest with the contents of this article.

\section{Funding sources/sponsors:}

Self-funded. 


\section{REFERENCES}

1. Yang S, Jee YJ, Ryu DM: Reconstruction of large oroantral defects using a pedicled buccal fat pad. Maxillofac Plast Reconstr Surg. 2018 Apr 5; 40(1):7.

2. Visscher SH, van Minnen B, Bos RR : Closure of oroantral communications: a review of the literature. J Oral Maxillofac Surg. 2010 Jun; 68(6):1384-91

3. Rehrmann A. A method of closure of oro-antral communications. Dtsch Zahnarzatl. Z . 1936, 39, 1163-1139.

4. Gibaly A, Mounir M, Mounir S; A combined staged novel approach for the reconstruction of persisting oro-antral fistulae defects utilizing tripled layer closure and open sinus lifting. E.D.J. Vol. 65, 3295:3303, October 2019.

5. Watzak G, Trepper, A, Zechner W, et al. Bony press-fit closure of oro-antral fistulas: a technique for pre-sinus lift repair and secondary closure. J Oral Maxillofac Surg. 2005 Sep;63(9):1288-94.

6. Ram H, Makadia H, Mehta G, et al. Use of Auricular Cartilage for Closure of Oroantral Fistula: A Prospective Clinical Study. J Maxillofac Oral Surg. . 2016 Sep;15(3):293-299.

7. Dym H, Wolf JC: Oroantral communication. Oral Maxillofac Surg Clin North Am. 2012 May; 24(2):239-47.

8. Del Fabbro M, Testori T, Franchetti L, et al. Systematic review of survival rates for implants placed in the grafted maxillary sinus. The International Journal of Periodontics \& Restorative Dentistry 2004; 24: 565-577.

9. Schmitt CM, Moest T, Lutz R, et al. Anorganic bovine bone (ABB) vs. autologous bone (A.B.) plus ABB in maxillary sinus grafting. A prospective nonrandomized clinical and histomorphometrical trial. Clin. Oral Impl. Res. 2015;26: 1043- 1050
10. Saleh EA, Issa IA: Closure of large oroantral fistulas using septal cartilage. Otolaryngol Head Neck Surg. 2013 Jun; 148(6):1048-50.

11. Abuabara A, Cortez AL, Passeri LA, de Moraes M, Moreira RW. Evaluation of different treatments for oroantral/ oronasal communications: experience of 112 cases. Int J Oral Maxillofac Surg. 2006 Feb; 35(2):155-8.

12. Lee BK: One-stage operation of large oroantral fistula closure, sinus lifting, and autogenous bone grafting for dental implant installation. Oral Surg Oral Med Oral Pathol Oral Radiol Endod. 2008 Jun; 105(6):707-13.

13. Ogunsalu C: A new surgical management for oro-antral communication: the resorbable guided tissue regeneration membrane, bone substitute sandwich technique. West Indian Med J. 2005 Sep; 54(4):261-3.

14. Hising P, Bolin A, Branting C. Reconstruction of severely resorbed alveolar crests with dental implants using a bovine bone mineral for augmentation. lnt J Oral Maxillofac Implants 2001; 16:90-97

15. Sohn DS, Lee JS, Ahn MR, et al. New bone formation in the maxillary sinus without bone grafts. Implant Dent.2008;17:321.

16. Sohn DS, Moon JW, Moon KN, et al. New bone formation in the maxillary sinus using only absorbable gelatin sponge. J Oral Maxillofac Surg.2010; 68: 1327.

17. Kilin Ç, A, Ataol, M, Dayi, E. Maxillary sinus lifting procedures using xenogeneic cortico-cancellous graft material with simultaneous implant placement: a 1- to 3-year follow-UP. Journal of Atatürk University Faculty of Dentistry 2018;26:1 